\title{
INFLUENCE OF TWIST ON SELECTED PROPERTIES OF MULTIFILAMENT YARN
}

\author{
Iva Mertová, Eva Moučková, Bohuslav Neckář, Monika Vyšanská \\ Technical University of Liberec, Faculty of Textile Engineering, Department of Technologies and Structures, \\ Liberec, The Czech Republic, Studentská 2, 46117 Liberec 1. Telephone: +420 48535 3274;
}

E-mail: eva.mouckova@tul.cz

\begin{abstract}
:
Owing to twisting of filament fiber bundle, the structure and consequently various parameters and properties of a fiber bundle are changed. The aim of the work is to verify the effect of multifilament yarn twist (or twist coefficient) on selected mechanical properties such as multifilament tenacity, breaking elongation, and coefficient of fiber stress utilization in the yarn. Furthermore, the influence of twist on structural parameters such as the angle of peripheral fibers, the packing density, and the substance cross-sectional area of fiber bundle is observed. Two multifilament yarns with different filament cross-section shape and material were used for the experiment. Experimentally obtained data was compared with the known model dependencies derived decades ago based on the helical model. It can be stated that multifilament yarn retraction can be predicted based on the angle of peripheral fibers using the Braschler's model. The coefficient of fiber stress utilization in the multifilament yarn determined experimentally corresponds with a theoretical curve, constructed according to Gégauff and Neckár̆, in the area of Koechlin's twist coefficient $\alpha>54 \mathrm{ktex}^{1 / 2} \mathrm{~m}^{-1}$. Results as well as possible causes of deviations of experimental data from the theoretical one are discussed in this work.
\end{abstract}

\section{Keywords:}

Multifilament yarn breaking tenacity, multifilament yarn breaking elongation, helix model, twist, packing density, yarn retraction

\section{Introduction}

Twisting of filament fiber bundle leads to a change in structure and, consequently, to a change in various parameters and characteristics of the fiber bundle. In addition to measurement conditions and material properties, a slope of fibers to the linear axis of multifilament yarn is also one of the most important factors affecting mechanical and physical properties of the fibers bundle (the shape of tensile stress-strain curve, tenacity, and breaking elongation of multifilament yarn). Knowledge of the behavior of twisted multifilament yarn (the fibrous bundle) is necessary for the prediction of similar twisted linear assemblies used, for example, for technical purposes (e.g., ropes). By twisting, not only the structure but also the mechanical properties of linear products are changed. The requirement for sufficient strength is essential in these technical applications. Therefore, the models related to structural characteristics of twisted multifilament yarns and their mechanical-physical properties are presented. Models and derived relationships have been chosen for their simplicity and ease application.

\section{Twisting of fibers bundle}

The continuous process of twisting of fibers bundle takes place under the action of twisting moment, which is derived by appropriate twisting system, and under the action of force during the yarn delivery. The effects of these forces lead to the fact that a mass point of fiber does rotational movement about its axis and simultaneously advance motion parallel to the axis of twisted fiber bundle. From the viewpoint of geometry, preconditions for the arrangement of fibers in a helical form are created [1]. The geometric arrangement of filaments (fibrils) in the structure of twisted fiber bundles can be described by a model of concentric helixes - the so-called ideally helical model (see Figure 1) [2], [3]. This model assumes the following: - The axes of all fibers have the shape of a helix with the same direction of rotation.

- Helixes of all fibers have one common axis, which is a multifilament yarn axis.

- The height of one coil of each helix is the same.

- The packing density is the same at all places inside the multifilament yarn.

We consider that peripheral fibers have the shape of helix with an angle of fiber slope to the multifilament yarn axis $\beta_{D}$ and height of one fiber coil is $1 / Z$. The quantity $Z$ expresses the yarn twist or the number of twists per unit length, and $D$ is the diameter of the yarn.

Number of authors, for example, Gégauff [4], Hearle [3], and Neckár [2] together with Das [5] and others [6-8], are dealing with these issues, in particular modeling of changes in mechanical properties.

Unrolled cylinder surface (the surface of multifilament yarn) forms a triangle. See Figure 1 from which equation (1) results:

$$
\operatorname{tg} \beta_{D}=\pi D Z=\frac{2 \sqrt{\pi} \alpha}{\sqrt{\mu \rho}}
$$




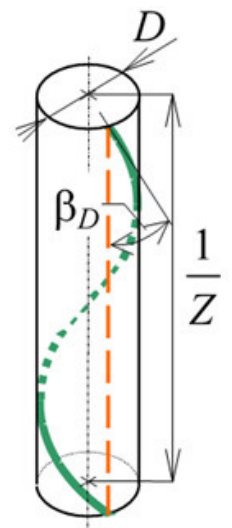

a

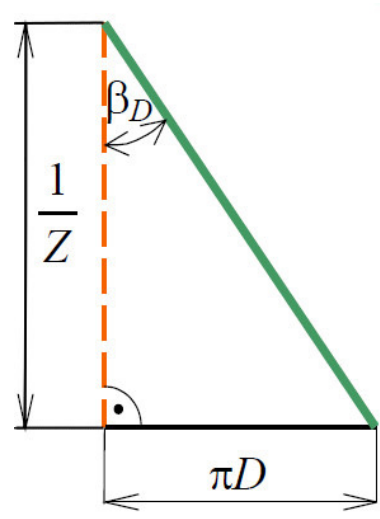

b

Figure 1. A peripheral fiber in helical model of multifilament yarn of diameter $D$ [10]. (a) One coil of a helical fiber on yarn surface and (b) Unrolled yarn surface

Here, $\beta_{D}$ denotes the angle of slope of peripheral fiber to the linear axis of twisted fiber bundle (shortly, the angle of peripheral fiber), $D$ is the diameter of cylindrical helix of peripheral fibers axis, $Z$ is the twist (strictly speaking, the number of twists per unit length of twisted fiber bundle), $\alpha$ is Koechlin's twist coefficient, $\rho$ is the fiber density, and $\mu$ is the packing density of fiber bundle.

The dependence between twist $Z$ and fineness $T$ of twisted fiber bundle was derived by Koechlin, see equation (2):

$$
Z=\frac{\alpha}{\sqrt{T}}
$$

Owing to twisting, the length of fiber bundle reduces. This shortening is expressed by the so-called yarn retraction $\delta$ (or also yarn twist take-up). The yarn retraction is thus given by the ratio of shortening of fiber bundle to its initial length, see equation (3).

$$
\delta=\frac{\text { Initial length-Length of twisted multifilament }}{\text { Initial length }}
$$

We can express the yarn twist take-up $\delta$ [\%] from the ideal helical model using equation (4).

$$
\delta=\frac{\sqrt{1+(\pi D Z)^{2}}-1}{\sqrt{1+(\pi D Z)^{2}}+1}
$$

Using equations (1) and (4), we obtain

$$
\delta=\frac{\sqrt{1+4 \pi \alpha^{2} /(\mu \rho)}-1}{\sqrt{1+4 \pi \alpha^{2} /(\mu \rho)}+1}
$$

By mathematical correction, based on the knowledge of yarn twist take-up $\delta$ and Koechlin's twist coefficient $\alpha$ for given material, we can express equation (6) for calculating packing density $\mu$ as

$$
\mu=\frac{\pi \alpha^{2}}{\rho} \frac{(1-\delta)^{2}}{\delta}
$$

According to the experience and known theoretical models, we expect that with the increasing number of twist per unit length of multifilament yarn, the yarn tenacity decreases, yarn breaking elongation (relative extension) increases, value of yarn retraction increases, and the related value of yarn count gets higher. Concurrently, yarn packing density and angle of peripheral fibers increase and multifilament yarn diameter changes.

The main objective of this work is to perform the experiment - twisting in originally non-twisted filament bundle, the measurement of selected parameters and characteristics for given level of multifilament yarn twist, and verification of assumptions. Obtained trends are compared with model's trends with the aim to present their validity and thus their possible utilization in next research connected with twisted multifilament yarns in various applications.

\section{Experiment and results}

Flat polyester (PES) and polypropylene (PP) multifilament yarns were used in the experiment. Their specification is mentioned in Table 1. Microscopic images of cross section of multifilament yarn are shown in Figure $2 a$ and $2 b$. Cross sections were made by a method of soft slices according to the internal standard [9].

The multifilament yarn was twisted using a twist tester. Before removing a sample from the jaws of twist tester, the twisted sample of multifilament yarn was fasten into a jig with collets to avoid yarn untwisting during subsequent handling with the yarn.

The angle between the axis of fiber on surface and the line parallel to the yarn axis $\beta^{\prime}$ (shortly, the angle in the axis of surface fibers) was measured using an image analysis. The demonstration of measurement is shown in Figure $3 \mathrm{a}$ and $3 \mathrm{~b}$. We carried out 300 measurements from every level of twist.

Strength and breaking extension of multifilament yarns were measured using tensile tester Instron under the following conditions: clamping length of $400 \mathrm{~mm}$, cross-beam speed of $280 \mathrm{~mm} \mathrm{~min}^{-1}$ (PES) and $360 \mathrm{~mm} \mathrm{~min}^{-1}$ (PP), and pre-tension of $0.5 \mathrm{cN} \mathrm{tex}{ }^{-1}$. We carried out 50 measurements from every level of twist. We also measured the strength and breaking extension of individual fibrils in multifilament yarns. These measurements were carried out under the same conditions as the measurements of multifilament yarns. Table $2 a$ and $2 \mathrm{~b}$ displays the resulting number of twists $(Z)$ of twisted 


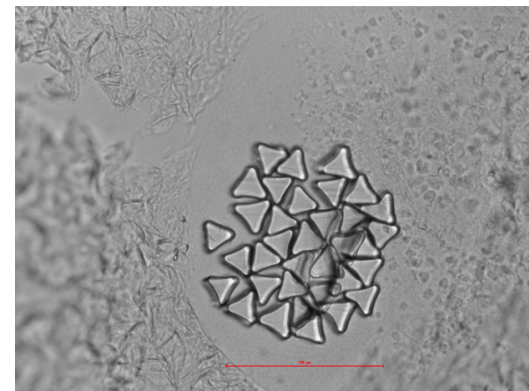

Figure 2a. Cross section of PES multifilament yarn

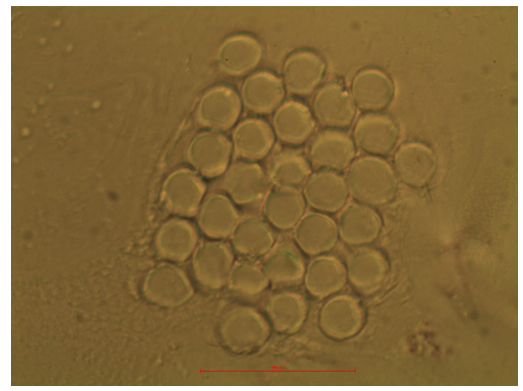

Figure 2b. Cross section of PP multifilament yarn

Table 1. Parameters of multifilament yarn used in the experiment

\begin{tabular}{|c|c|c|c|}
\hline Material & $\begin{array}{c}\text { Nominal count of multifilament } \\
\text { yarn } \mathbf{T}_{\text {nom }} \text { [tex] }\end{array}$ & Number of fibril & Fiber density $\boldsymbol{\rho}\left[\mathbf{k g} \cdot \mathbf{m}^{-\mathbf{3}}\right.$ ] \\
\hline $\mathbf{1 0 0} \%$ PES & 10 & 36 & 1,380 \\
\hline $\mathbf{1 0 0} \%$ PP & 16.7 & 32 & 910 \\
\hline
\end{tabular}

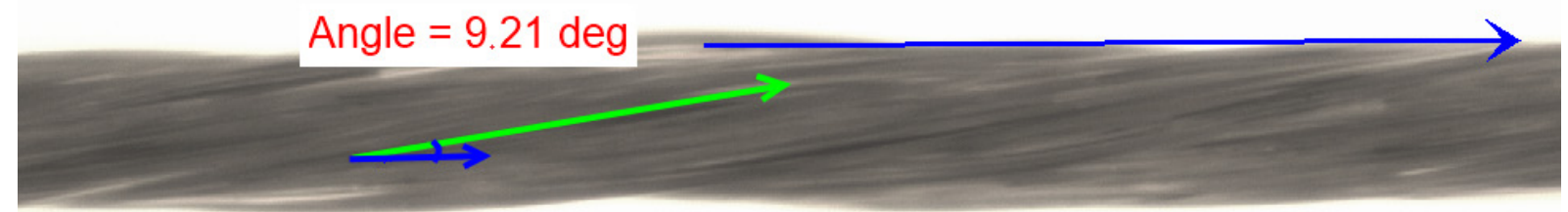

Figure 3a. Demonstration of the measurement of angle in the axis of peripheral fibers $\beta^{\prime}$, PES multifilament yarn, $Z=500 \mathrm{~m}^{-1}$

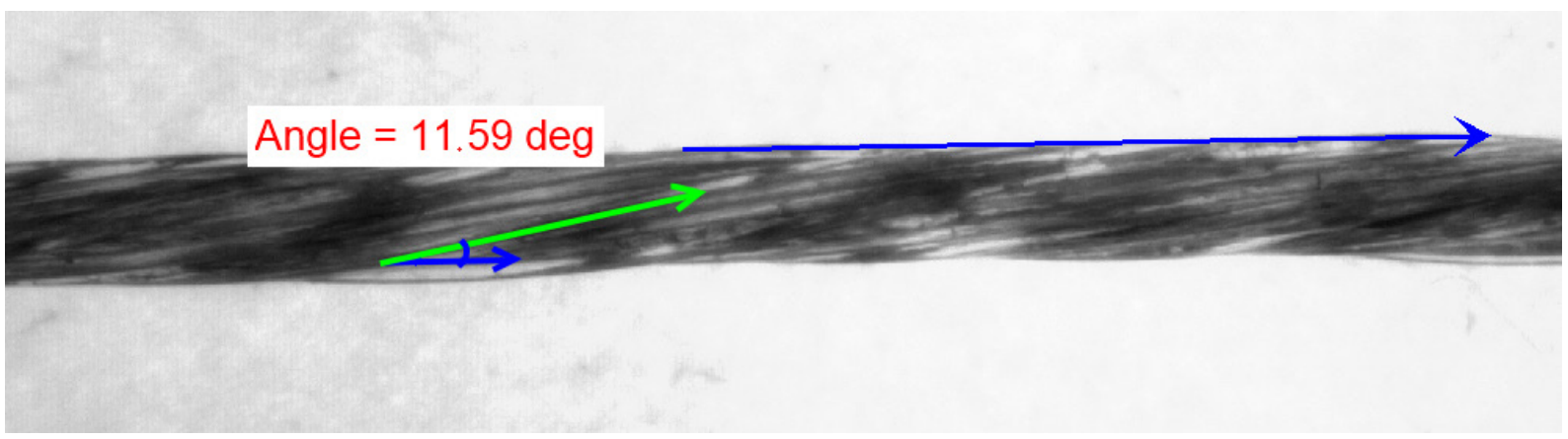

Figure 3b. Demonstration of the measurement of angle in the axis of peripheral fibers $\beta^{\prime}$, PP multifilament yarn, $Z=408 \mathrm{~m}^{-1}$

multifilament yarns together with values of respective yarn twist take-up $\left(\delta_{E}\right)$, actual twisted yarn counts $(T)$, calculated Koechlin's twist coefficient $(\alpha)$, tenacity $(\sigma)$, and breaking elongation $(\varepsilon)$ of individual fibrils and multifilament yarns. For clarity, only average values of observed parameters and properties are shown in the table. The dependence of the angle in the axes of peripheral fibers $\beta^{\prime}$ on the multifilament twist coefficient is shown in Figure 5.

\section{Discussion of results}

\subsection{Substance cross-sectional area}

As mentioned earlier, by twisting the bundle of parallel fibers, the bundle is shortened. Thus, the value of twisted bundle count $T$ should be higher compared with the value of nontwisted bundle count $T_{0}$ according to equation (7). If we relate twisted multifilament yarn count $T$ to the fiber density $\rho$, we obtain substance cross-sectional area $S$ of multifilament yarn, thus the summation of cross-sectional area of fibers in the yarn cross section. The substance cross-sectional area of yarn is given by equation (8).

$$
\begin{aligned}
& T_{0}=T(1-\delta) \\
& S=T / \rho
\end{aligned}
$$

The comparison of substance cross-sectional area $S$ of both experimental multifilament yarns in dependence on Koechlin's twist coefficient is mentioned in Figure 4. We can see presumed increase in substance cross-sectional area of 
Table 2a. PES multifilament yarn - results of observed parameters and properties

\begin{tabular}{|c|c|c|c|c|c|}
\hline $\begin{array}{l}\text { Twist } \\
\mathrm{Z}\left[\mathrm{m}^{-1}\right]\end{array}$ & $\begin{array}{l}\text { Yarn twist take- } \\
\text { up } \delta_{E}[\%]\end{array}$ & $\begin{array}{c}\text { Multifilament } \\
\text { count } \\
T \text { [tex] }\end{array}$ & $\begin{array}{c}\text { Koechlin's twist } \\
\text { coefficient } \\
\alpha\left[k^{1 / 2} \mathbf{x}^{1 / 2} \cdot \mathrm{m}^{-1}\right]\end{array}$ & $\begin{array}{l}\text { Tenacity } \\
\sigma[\mathrm{cN} / \mathrm{tex}]\end{array}$ & $\begin{array}{c}\text { Breaking } \\
\text { elongation } \\
\varepsilon[\%]\end{array}$ \\
\hline $0^{*}$ & - & 0.318 & - & 41.4 & 24.791 \\
\hline 0 & - & 11.436 & 0 & 32.492 & 25.974 \\
\hline 100 & 0.05 & 11.442 & 10.7 & 33.272 & 27.472 \\
\hline 200 & 0.2 & 11.459 & 21.4 & 33.845 & 28.474 \\
\hline 300 & 0.4 & 11.482 & 32.1 & 35.053 & 31.230 \\
\hline 400 & 0.68 & 11.514 & 42.9 & 34.905 & 32.057 \\
\hline 500 & 1.022 & 11.554 & 53.7 & 35.811 & 34.749 \\
\hline 600 & 1.422 & 11.601 & 64.6 & 35.253 & 34.947 \\
\hline 700 & 1.978 & 11.666 & 75.6 & 35.510 & 36.008 \\
\hline 800 & 2.502 & 11.729 & 86.6 & 35.362 & 36.717 \\
\hline 900 & 2.92 & 11.78 & 97.7 & 34.896 & 38.043 \\
\hline 1,000 & 3.85 & 11.894 & 109.1 & 34.526 & 37.848 \\
\hline 1,500 & 8.108 & 12.445 & 167.3 & 29.893 & 38.393 \\
\hline 2,000 & 13.135 & 13.165 & 229.5 & 26.102 & 40.008 \\
\hline
\end{tabular}

Note: 0 * represents the results of individual fibrils measurements

Table 2b. PES multifilament yarn - results of observed parameters and properties

\begin{tabular}{|c|c|c|c|c|c|}
\hline $\begin{array}{l}\text { Twist } \\
\mathrm{Z}\left[\mathrm{m}^{-1}\right]\end{array}$ & $\begin{array}{l}\text { Yarn twist take- } \\
\text { up } \delta_{E}[\%]\end{array}$ & $\begin{array}{c}\text { Multifilament } \\
\text { count } \\
\mathrm{T}[\text { tex] }\end{array}$ & $\begin{array}{c}\text { Koechlin's twist } \\
\text { coefficient } \\
\alpha\left[k^{1 / 2} x^{1 / 2} \cdot m^{-1}\right]\end{array}$ & $\begin{array}{c}\text { Tenacity } \\
\sigma[c N / t e x]\end{array}$ & $\begin{array}{c}\text { Breaking } \\
\text { elongation } \\
\varepsilon[\%]\end{array}$ \\
\hline $0 *$ & - & 0.536 & - & 45.029 & 57.004 \\
\hline 0 & - & 17.163 & 0 & 42.229 & 51.746 \\
\hline 82 & 0.091 & 17.179 & 10.7 & 41.790 & 46.263 \\
\hline 163 & 0.251 & 17.206 & 21.4 & 41.760 & 47.209 \\
\hline 245 & 0.567 & 17.261 & 32.2 & 42.239 & 49.331 \\
\hline 326 & 1.022 & 17.340 & 43 & 41.500 & 48.944 \\
\hline 408 & 1.496 & 17.427 & 53.8 & 41.596 & 54.630 \\
\hline 490 & 2.034 & 17.519 & 64.8 & 40.667 & 54.290 \\
\hline 571 & 2.688 & 17.637 & 75.9 & 40.432 & 55.165 \\
\hline 653 & 3.549 & 17.794 & 87.1 & 40.018 & 56.568 \\
\hline 734 & 4.477 & 17.967 & 98.4 & 39.294 & 51.516 \\
\hline 816 & 5.492 & 18.160 & 110 & 39.081 & 56.012 \\
\hline 1224 & 11.953 & 19.493 & 171 & 35.144 & 52.035 \\
\hline 1632 & 19.588 & 21.344 & 238.5 & 29.379 & 46.736 \\
\hline
\end{tabular}

Note: 0 * represents the results of individual fibrils measurements 
multifilament yarns. With the increasing twist number, the yarn length gets shorter, thus yarn mass per unit length is higher. The multifilament yarns get coarser. Owing to the constant density of the material (of the given multifilament yarn), the substance cross-sectional area increases too, see relation (8). The increase in substance cross-sectional area was confirmed in both cases independently of the shape of fibrils and kind of material. Therefore, yarn count can be expressed geometrically through the yarn cross-sectional area. The PES multifilament yarn has a smaller geometrically expressed yarn count $S$ compared with PP multifilament yarn.

\subsection{Angle of peripheral fibers}

Using the image analysis, we measured the angle in the axis of surface fibers $\beta^{\prime}$ from the longitudinal views of multifilament yarn as mentioned earlier. For this angle, equation (9) is valid:

$$
\operatorname{tg} \beta^{\prime}=\pi(D-d) Z
$$

(9) The experiment confirmed that with the increasing Koechlin's twist coefficient, both the angle measured in the axis of surface

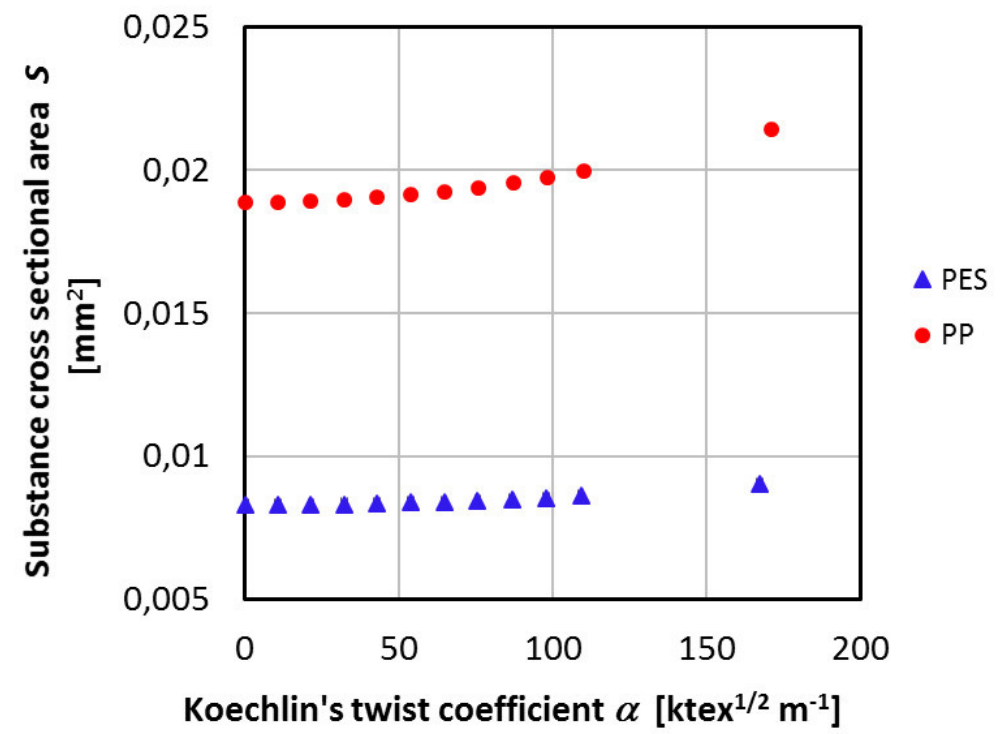

Figure 4. Influence of yarn substance cross-sectional area on Koechlin's twist coefficient

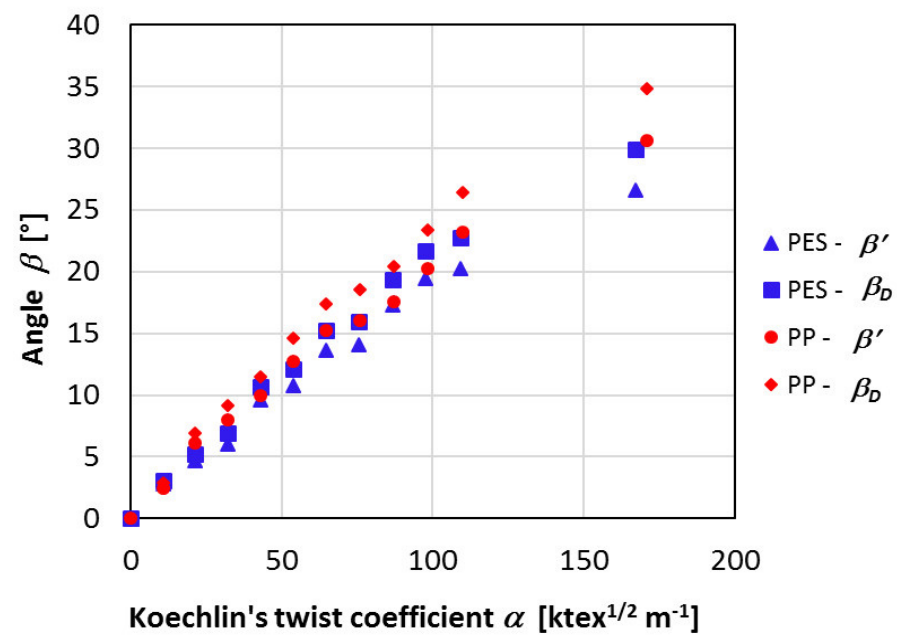

Figure 5. Dependence of the angle of surface fibers slope in the multifilament on Koechlin's twist multiplier 
fibers $\beta^{\prime}$ and the angle of peripheral fibers $\beta_{D}$ in multifilament yarn increase almost linearly. This dependence was assumed. Considering the constant multifilament yarn count and the increasing value of Koechlin's twist coefficient, the yarn twist level also increases according to relation (2). In the term of multifilament yarn structure, the twist is considered to be the number of coils per unit of length. Thus, as shown in Figure $1 \mathrm{~b}$, with the increasing twist, assuming the helical model, the angle of slope of peripheral fiber $\beta_{D}$ to the linear axis of fiber bundle increases.

Deviations from linearity at higher values of twist coefficients are probably caused due to the saturated twist and formation of twist of the second order, that is, an "overtwisting" of multifilament yarn. Figure 6 demonstrates the twist of the second order.

\subsection{Multifilament yarn retraction}

Based on knowledge of angle of peripheral fiber $\beta_{D}$, we can predict the yarn retraction $\delta_{p}$ using equation (12), which was derived by Braschler. This equation was presented, for example, in work [10].

$$
\delta_{P}=\operatorname{tg}^{2}\left(\frac{\beta_{D}}{2}\right)
$$

The comparison of experimentally determined multifilament yarn twist take-up $\delta_{E}$ and predicted multifilament yarn twist take-up $\delta_{T}$ (using the angle $\beta_{D}$ ) is shown in Figure 7 .

The experiment confirmed the validity of theoretical model. In both cases, we can see statistically significant correlation coefficients (see Table 3 ) showing a linear relationship between the experimentally determined values of yarn twist take-up $\delta_{E}$ and predicted values of yarn twist take-up $\delta_{T}$ calculated using the angle of peripheral fibers $\beta_{D}$. For verification of the significance of correlation coefficient $(R)$, we used corresponding significance test [13]. The basic hypothesis $R$ $=0$ was tested against various alternative hypothesis. If the calculated test statistics is higher than corresponding $95 \%$ quantile of Student's distribution, the basic hypothesis is rejected and random variables are correlated (assuming a twodimensional normal distribution). The significant correlation coefficient between experimental and theoretical twist take-up verifies the fact that with the growing multifilament yarn twist level, assuming the helical model, the angle of peripheral

PES multifilament, $Z=2000 / \mathrm{m}$

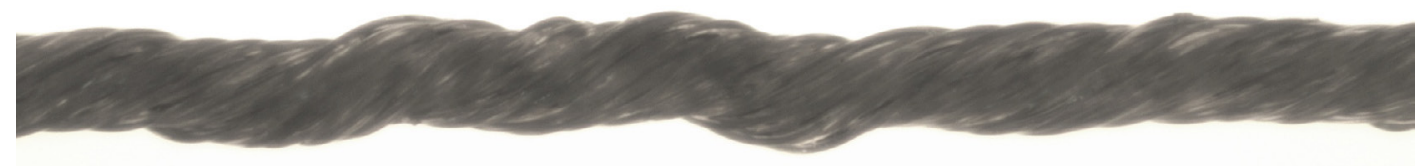

PP multifilament, $Z=1600 / m$

Figure 6. Illustration of twist of the second order

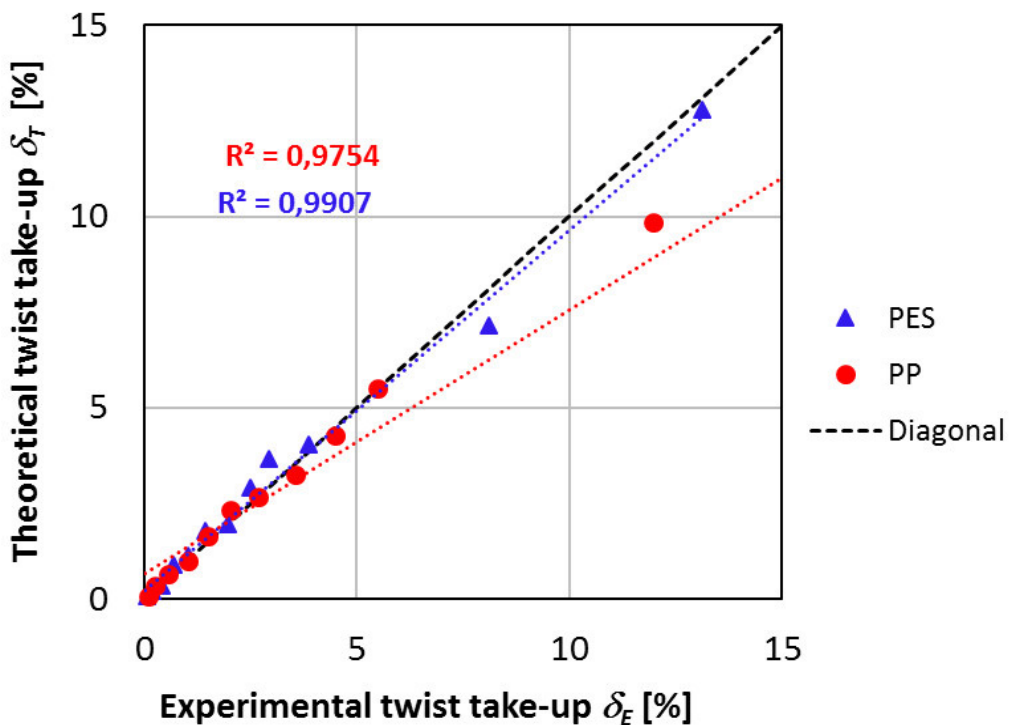

Figure 7. Dependence of experimental $\delta_{E}$ and theoretical $\delta_{T}$ multifilament yarn twist take-up 
Table 3. Correlation coefficient between experimental and predicted values of multifilament yarn twist take-up

\begin{tabular}{|c|c|c|c|c|}
\hline Material & Twist take-up & Correlation coefficient R & Test statistics & $\begin{array}{c}\text { 95\% quantile of } \\
\text { Student's distribution }\end{array}$ \\
\hline PES & $\delta_{E}$ versus $\delta_{T}$ & 0.995 & 32.677 & 1.181 \\
\hline PP & $\delta_{E}$ versus $\delta_{T}$ & 0.987 & 19.933 & 1.181 \\
\hline
\end{tabular}

fibers to the axis of yarn increases and thus yarn twist take-up increased too.

\subsection{Multifilament yarn packing density}

Based on the knowledge of yarn twist take-up (experimental values), we can predict the course of multifilament yarn packing density on Koechlin's twist coefficient using equation (6). Figure 8 displays this course. The graph shows the increase in the multifilament yarn packing density with increasing Koechlin's twist coefficient. During twisting, individual filaments are closer to each other and thus multifilament packing density increased. But when reaching higher values of twist coefficient (approximately $\alpha=75 \mathrm{ktex}^{1 / 2} \mathrm{~m}^{-1}$ and higher), the values of multifilament yarn packing density gets stabilized. Fibrils can no longer be closer to each other. When being nearly constant, the level of packing density takes the value of $\mu=0.68$. The value corresponds with the values of packing density presented in work [2]. From the results, it is clear that the proportion of total volume of multifilament yarn filled with the volume of fibrils does not reach the limiting packing density $(\mu=0.907$ [2]) even in the case of the maximum observed twist level. This is probably due to the fact that the fibrils in the multifilament yarn are not placed absolutely parallel to each other. The value of limiting packing density was determined in the case of a hexagonal tight structure, assuming the parallelism of fibrils. This assumption cannot be met due to the twist.

\subsection{Tenacity}

Average values of multifilament yarn tenacity $(\sigma)$ with relevant confidence intervals (on the confidence level of $0.05 \%$ ) depending on Koechlin's twist coefficient are plotted in Figure 9. From the figure, it is seen that the dependence of multifilament yarn tenacity on Koechlin's twist multiplier is influenced by the type of material and cross-sectional shape of individual fibrils. Tenacity of PES multifilament yarn is increasing up to twist multiplier $\alpha=54 \mathrm{ktex}^{1 / 2} \mathrm{~m}^{-1}$, then the tenacity is decreasing. The reason of tenacity increasing in the area of relatively small twist multipliers is probably the action of the cohesion forces. Cohesion forces increase with increasing twist value. The surge of these forces is involved with triangular cross-sectional shape of individual fibers. When these fibers get closer to each other during twisting, the friction between individual fibers is increasing.

The tenacity of PP multifilament yarn varies slightly with increasing twist factor up to twist multiplier $\alpha=53 \mathrm{ktex}^{1 / 2} \mathrm{~m}^{-1}$. In this case, the cohesive forces are not applied in the same extend as in the case of triangular cross section of filament. The dependence between multifilament tenacity and twist factor may be influenced by crimping of individual fibers in the area of small twist multipliers.

Lower utilization of tensile forces of individual fibers decreases the tenacity of both multifilament yarns. The coefficient of fiber stress utilization in the multifilament yarn decreases with increasing twist factor value by reason of higher slope of fibers to the multifilament axis. We can calculate coefficient of fiber stress utilization in multifilament yarn (fiber bundle) $\varphi$ based on the experimental values according to equation (13) and based on the model predicting equation (14). This model was derived by Gégauff [4] and then modified by Neckár̆ [2]. The assumptions of this model are the following: individual fibrils are straight; there

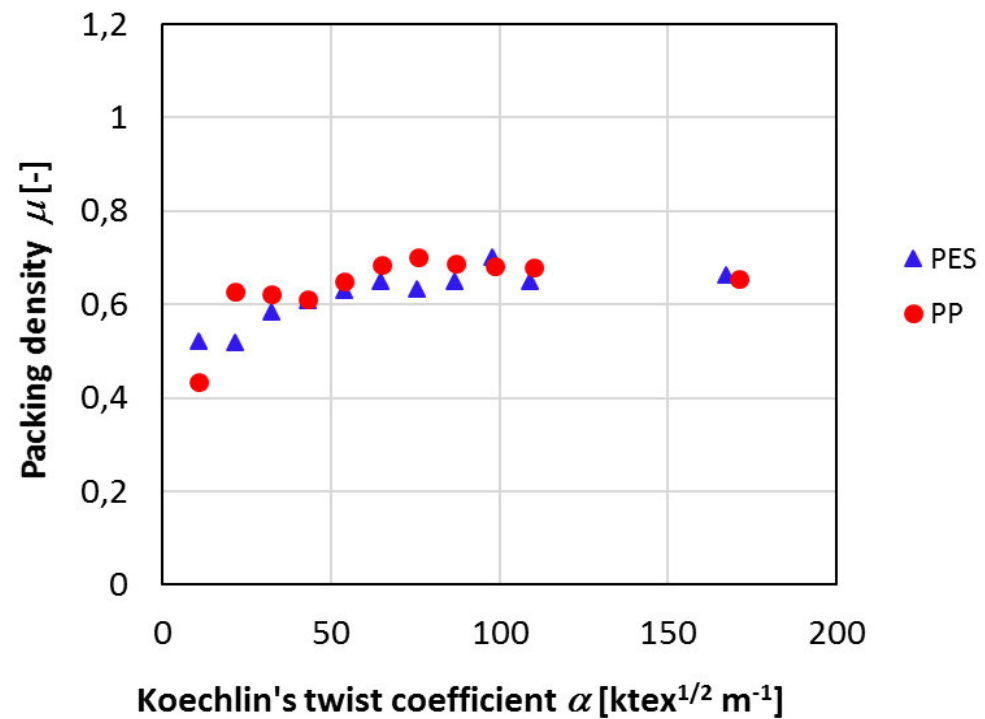

Figure 8. Dependence of multifilament packing density on twist coefficient 


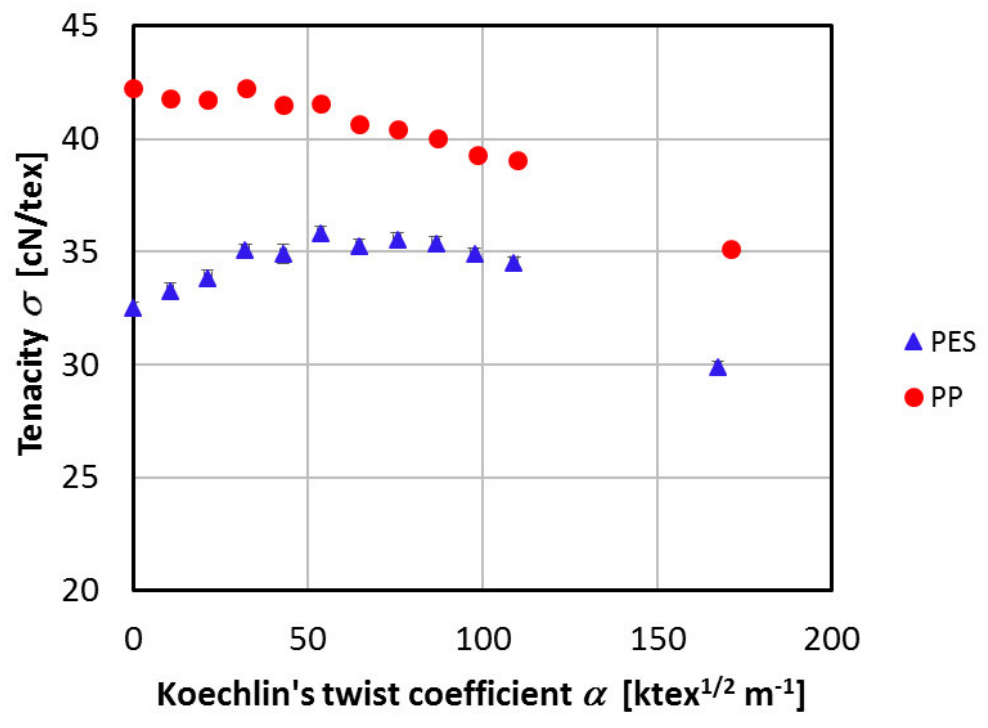

Figure 9. Dependence of multifilament yarn tenacity on Koechlin's twist multiplier

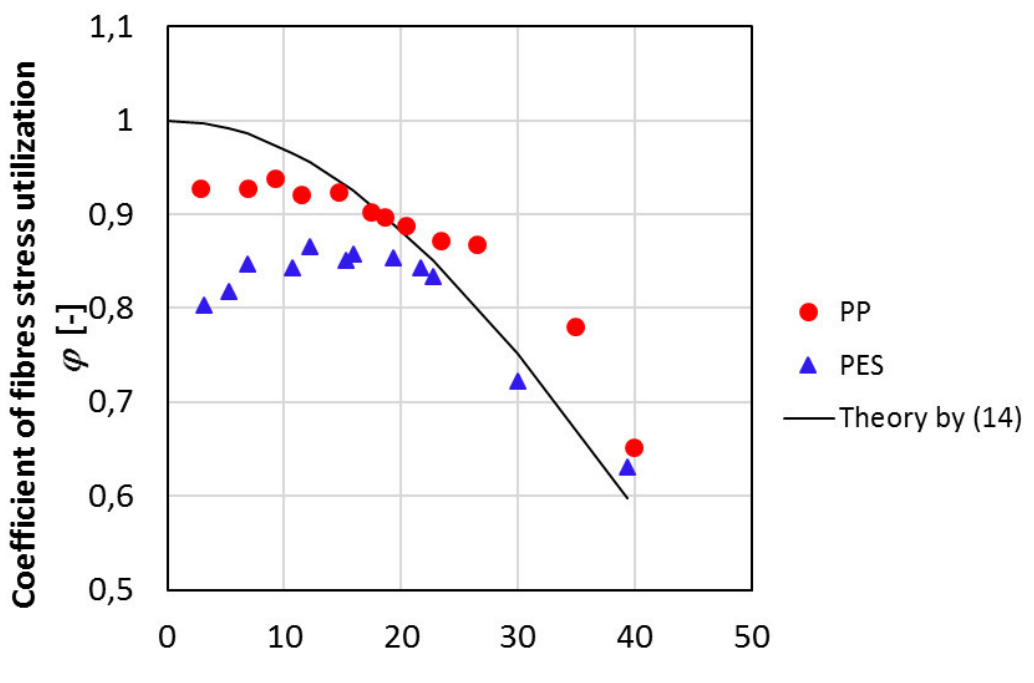

Angle of peripheral fibre $\beta_{D}\left[^{\circ}\right]$

Figure 10. Dependence of coefficient of fiber stress utilization in twisted multifilament yarn on angle of peripheral fiber

is no interaction between individual fibers during fiber bundle tension; and stress-strain curves of fibers are linear. More general solution of this problem is mentioned in [12].

$$
\varphi=\sigma / \sigma^{*}
$$

where $\sigma$ is the tenacity of twisted fiber bundle and $\sigma^{*}$ is the tenacity of individual fibers.

$$
\varphi=(1+\eta) \cos ^{2} \beta_{D}+\eta \frac{\ln \cos ^{2} \beta_{D}}{\operatorname{tg}^{2} \beta_{D}}
$$

where $\beta_{D}$ is the angle of peripheral fiber and $\eta$ is Poisson's contraction ratio $[-]$.

It is necessary to know value of Poisson's ratio for the evaluation of coefficient of fiber stress utilization. But it is problematic to determine this constant. In case if the value is unknown, it is generally set to $\eta=0.5$. The multifilament yarn volume is not changed with this ratio value during small tension deformations [2].
Coefficients of fiber stress utilization in the multifilament yarn calculated according to equations (13) and (14) are presented in Figure 10. The Poisson's contraction ratio $\eta=0$ was used for the calculation of $\varphi$ based on equation (14). The dependence of experimental and theoretical coefficient of fiber stress utilization in multifilament yarn on the angle of peripheral fiber (see Figure 10) corresponds to results presented in works [2] and [3]. From Figure 10, it is seen that the experimentally determined coefficient of fiber stress utilization of PES fibers is lower than the predicted one in the area of small angles (to $\beta_{D}=12^{\circ}$ ). The value of coefficient has increasing tendency to this angle. The reason is connected probably with different properties of individual fibers - crimping of fibers and variability of elongation at break. So the average fiber tenacity is higher than the tenacity of fiber bundle (the multifilament yarn). The crimping of fibers given by production technology are not identical; consequently, initial lengths of individual fibers are not identical. All fibers in the fiber bundle - multifilament yarn - are loaded non-uniformly. This fact can be the cause of reduction of fiber stress utilization in the multifilament yarn. The twisting 
of multifilament yarn is processed under stress. Crimped fibers are straightened and interacted during this process in the area of small angles of peripheral fibers. It can lead to the increase in cohesive forces and the increase in coefficient of fiber stress utilization in the multifilament yarn. Other reason can be the clamping (initial) length [11] or a modification of multifilament diameter during twisting.

The coefficient of fiber stress utilization in PP multifilament yarn is varying slightly around the value $\varphi=0.928$ to the value of angle of peripheral fiber ca. $\beta_{D}=14^{\circ}$. In this case, the mutual interaction can be affected by different shapes of fiber cross section or by crimping of individual fibers.

Experimental curves of coefficient of fiber stress utilization in both multifilament yarns correspond with theoretical curves in the area of angles higher than $12^{\circ}$ in the case of PES multifilament or $15^{\circ}$ in the case of PP multifilament yarn. In this area, fiber stress utilization decreases with increasing twist factor, because magnitude of the component of axial force in the fiber is lowering in the direction of multifilament axis.

The value of fiber stress utilization of PP fibers is higher than that in the case of PES fibers because of the higher tenacity and breaking elongation of the first one.

From Figure 10, it is evident that the experimental values of PP fiber utilization in the multifilament are higher than the predicted values in the area of ultra-high angles. This fact can be effected by distortion of multifilament, thus by reaching the saturated twist and twist of the second order with combination of very high breaking strain variability. The saturated twist is a limit case; it is really observed experimentally; in our experiment, the saturated twist corresponds to the angle $\beta_{D}$ from $25^{\circ}$ to $35^{\circ}$ in the case of PP multifilament yarn. The axial asymmetry of twisted multifilament yarn is a probable reason of this phenomenon. If we give higher twist than the saturated twist to the yarn, the yarn is not able to absorb it into its structure and then some coils are placed at the surface as coils of twist of second order.

\subsection{Breaking elongation}

Average values of breaking elongation $\varepsilon$ together with their $95 \%$ confidence interval depending on Koechlin's twist multiplier are plotted in Figure 11.

The breaking elongation has increasing tendency with increasing value of twist factor to the value $\alpha=100 \mathrm{ktex}^{1 / 2} \mathrm{~m}^{-1}$ (87 in PP multifilament). The hyper supply of material on given length owing to twisting increases this breaking elongation. In the area of higher values of Koechlin's twist factor, the breaking elongation is practically constant because of the saturated twist and the twist of second order, see Figure 6 . The variable course of PP multifilament breaking elongation is probably caused by the influence of high variability of tensile characteristics of individual fibers. The breaking elongation of PP multifilament is higher compared with PES multifilament because of the higher elongation of PP individual fibers.

We can predict axial strain of fiber bundle based on the measured values of breaking strain of untwisted fiber bundle and values of angle of peripheral fibers using Gégauff's model [4]. This model was modified by Neckář [2] for different values of Poisson's ratio, see equation (15). The simplified assumptions of this model are helical structure of all fibers with the same direction of rotation in the fiber bundle, no deformation during twisting, and no migration of fibers.

$$
\varepsilon_{l}=\varepsilon_{a}\left(\cos ^{2} \beta-\eta \sin ^{2} \beta\right)
$$

where $\varepsilon_{a}$ denotes the relative breaking elongation of twisted fiber bundle and $\varepsilon$, is the relative breaking elongation of nontwisted fiber bundle.

Predicted and experimental values of breaking elongation were compared mutually using correlation coefficients. Table 4 illustrates the observed correlation coefficients between experimental and predicted values. Correlation coefficients are statistically significant. Predicted values of breaking elongation were calculated according to equation (15) with Poisson's ratio

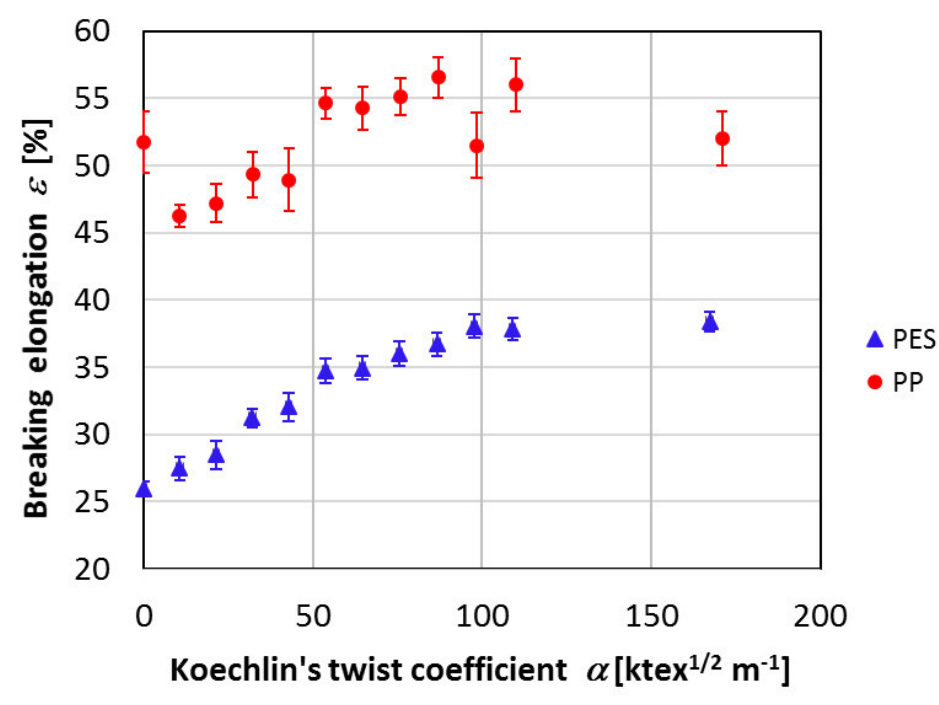

Figure 11. Dependence of multifilament yarn breaking elongation on Koechlin's twist multiplier 
$\eta=0$. Breaking elongations of yarn with very high twist $(Z>$ $900 \mathrm{~m}^{-1}$ in case of PP multifilament, $Z>1,500 \mathrm{~m}^{-1}$ in case of PES) were not included into the calculation of correlation coefficient. Using these, very high twist factors leads to the twist of second order, which does not correspond with helical model assumptions.

The comparison of experimental and predicted values of breaking strain of twisted multifilament yarns depending on the angle of peripheral fibers is visible in Figure 11. Theoretical values of breaking strain were calculated with Poisson's ratio $\eta=0$. The theoretical model supposes that the dependence between breaking strain and the angle of peripheral fiber has increasing trend. It is evident from the diagram in Figure 12, and also results presented in Table 4 acknowledge the model assumption.

Lower correlation coefficient between experimental and theoretical breaking strain in the case of PP multifilament yarn is due to higher variability of breaking elongation of multifilament. Other influence can be higher migration of PP fibrils because of the circular cross section of PP fibers.

\section{Conclusion}

The influence of twist coefficient on selected mechanicalphysical properties (tenacity and breaking strain) and structural characteristics (angle of peripheral fibers, packing density, and substance cross-sectional area) of multifilament yarn were analyzed. For the experimental part, we used two different multifilament yarns with different cross-sectional shape and material (PP and PES). Experimentally obtained data was compared with well-known model dependences.

It was confirmed that with increasing twist, the angle of peripheral fibers $\beta_{D}$ and the angle between the axis of fiber on surface and the line parallel to the yarn axis $\beta^{\prime}$ are increasing too. There is shortening (the twist take-up) of the multifilament yarn because of twisting. It leads to increasing in yarn count value, expressed by the substance cross-sectional area of multifilament yarn.

Experimentally obtained values of yarn twist take-up correspond with predicted values calculated according by Brashler's model based on the knowledge of angle of peripheral fibers.

The equation for prediction of packing density derived from the helical model was verified. Predicted values of packing density in the area of lower twist factors (approximately to the level $\alpha$ $=75 \mathrm{ktex}^{1 / 2} \mathrm{~m}^{-1}$ ) go up, crossing this level the packing density becomes constant and takes the value of about $\mu=0.68$.

It has been assumed that tenacity of both experimental multifilament yarns is decreasing with increasing twist factor. This fact was recorded from the twist factor level $\alpha=54 \mathrm{ktex}^{1 / 2}$ $\mathrm{m}^{-1}$. This value of twist factor corresponds to the angle of peripheral fibers: $10.8^{\circ}$ in the case of PES multifilament yarn and $12.77^{\circ}$ in the case of PP multifilament yarn.

The coefficient of fiber stress utilization was established experimentally and theoretically according to the model by Gégauff and Neckár. The behavior of coefficient of fiber stress utilization confirmed previously assumed results. The

Table 4. Correlation coefficient between experimental and theoretical breaking elongation of multifilament yarn

\begin{tabular}{|c|c|c|c|c|}
\hline Material & $\mathbf{\eta}$ & Correlation coefficient $\mathbf{R}$ & Test statistics & $\begin{array}{c}\text { 95\% quantile of Student's } \\
\text { distribution }\end{array}$ \\
\cline { 1 - 3 } PP & \multirow{2}{*}{0} & 0.738 & 3.456 & 1.86 \\
\cline { 1 - 4 } PES & & 0.908 & 6.843 & 1.86 \\
\hline
\end{tabular}

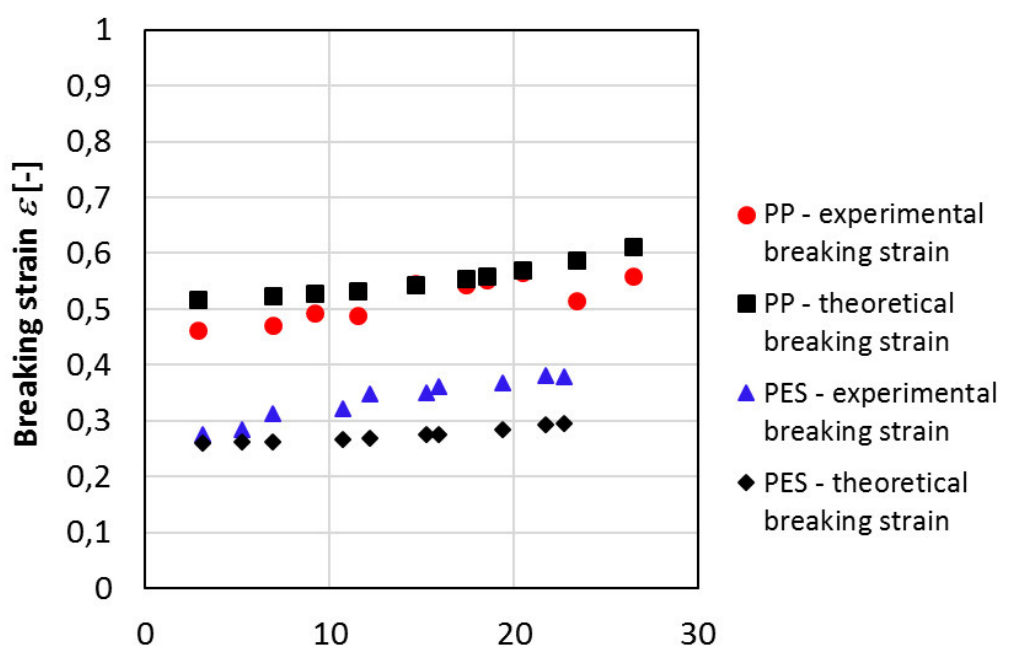

Angle of pheripheral fibre $\beta_{D}\left[^{\circ}\right]$

Figure 12. Comparison of experimental and theoretical breaking strain 
coefficient of fiber stress utilization in the area of small angles (to $\beta_{D}=12^{\circ}$ ) is influenced by the number of factors, whose mathematical description is difficult. The shape of fiber cross section, crimping of individual fibers, variability of elongation at break of individual fibers, clamping (initial) length, and modification of multifilament diameter during twisting belongs among these factors. Experimental curves of coefficient of fiber stress utilization $\varphi$ in both multifilament yarns correspond with theoretical curves in the area of twist factor, $\alpha>54 \mathrm{ktex}^{1 / 2} \mathrm{~m}^{-1}$. In this area, fiber stress utilization is decreasing with increasing twist factor. The experimental data of $\varphi$ corresponds with predicted data using the Poisson's contraction ratio $\eta=0$.

The breaking elongation has increasing tendency with increasing value of twist factor to the value $\alpha=100 \mathrm{ktex}^{1 / 2} \mathrm{~m}^{-1}$ (87 in PP multifilament). Owing to twisting, the hyper supply of material on given length increases this breaking elongation. In the area of higher values of Koechlin's twist factor, the breaking elongation is practically constant when increasing twist factor. It is due to the saturated twist and the twist of second order. The multifilament yarn is deformed.

It has been confirmed that experimental dependence of breaking elongation on the angle of peripheral fibers statistically correlates with predicted values according to the model by Gégauff and Neckár. The theoretical model supposes that the dependence of breaking strain and angle of peripheral fiber has increasing trend because of the twisting of multifilament yarn.

From the experiment it results that inner structure can be described by the helical model. Predicted relations based on the helical model, derived decades ago, are valid nowadays also, and they can be used in next research connected with twisted multifilament yarns in various applications. This work also opens up more space for research of multifilament yarns behavior in areas of low twist multipliers.

\section{Acknowledgment}

This work is supported under Student Grant Scheme (SGS 21196) by Technical University of Liberec, Czech Republic.

\section{References}

[1] Ursiny, P. (1984). Machines and Technology of Spinning in Czech. VŠST Liberec (Liberec).

[2] Neckář, B. (1990). Yarn-Forming, structure and properties. SNTL Prague (Prague).

[3] Hearle, J. W. S., Grosberg, P. and Backer, S. (1969). Structural Mechanics of Fibers, Yarns and Fabrics. WileyInterscience (New York).

[4] Gegauff, M. C. (1907). Strength and elasticity of cotton threads. Bulletin de la Société Industrielle de Mulhouse, 77, pp. $153-176$.

[5] Neckár̆, B., Das, D. (2012). Theory of structure and mechanics of fiber assemblies. (1 edition). Woodhead Publishing India (New Delhi).

[6] Jones, J. E. Haddad, G. N. and Sutton, J.N. (1971). Tensile Characteristics of Twisted Continuous-Filament Glass Yarns. Textile Research Journal. Vol. 41, Issue 11, pp. 900 $-904$.

[7] Kilby, W. F. (1964). The Mechanical Properties of Twisted Continuous Filament Yarns. The Journal of the Textile Institute, Volume 55, Issue 12, pp. T589-T632.

[8] Treloar, L. R. G. and Riding, G. A. (1963). Theory of Stress-Strain Properties of Continuous-Filament Yarns. The Journal of the Textile Institute, Volume 54, Issue 4, pp.T156-T170.

[9] Internal Standard No. 46-108-01/01 Recommended procedure for preparation of samples. Soft and hard sections (slices). Technical University of Liberec, Faculty of Textile Engineering, Textile Research Center.

[10] Neckar, B. (2014). Weaves Structure and Properties. Lectures. Retrieved June, 25, 2016. Web site: http://www. ktt.tul.cz/.

[11] Das, D. (2005). Yarn strength as a stochastic process. Dissertation work. Technical University of Liberec (Liberec).

[12] Neckar, B., Das, D. (2016): Tensile behavior of staple fiber yarns part l: theoretical models. The Journal of the Textile Institute, DOI: 10.1080/00405000.2016.1204899.

[13] Meloun, M., Militky, J. (1994): Statistical treatment of experimental data. PLUS, s.r.o. (Prague) 